\title{
TIC PARA LA EDUCACIÓN: SISTEMA ADAPTATIVO BASADO EN MECANISMOS DE APRENDIZAJE AUTOMÁTICO PARA LA APROPIACIÓN DE TECNOLOGÍAS EN ESTUDIANTES DE EDUCACIÓN MEDIA
}

ICT for education: adaptive system based on automatic learning mechanisms for the use of technologies in students*

Andrea Otero**, Wilfred Rivera ${ }^{* * *}$, Camilo Pedraza**** y José Raúl Canay $^{* * * * *}$

\section{RESUMEN}

La investigación presenta un enfoque basado en analítica de datos y mecanismos de aprendizaje automático integrado a una de las plataformas digitales de aprendizaje más usadas en el mundo (open EDX) como aporte al mejoramiento de los procesos de aprendizaje en estudiantes de educación media de municipios de Colombia. Metodológicamente con base en Creswell y Plano Clark (2007) y Clements et al. (2017), se construyó un sistema que posibilita la recomendación de contenidos educativos adecuados a las características individuales de estudiantes teniendo en cuenta las limitaciones en el uso y apropiación social de las tecnologías en las instituciones educativas, se realizaron entrevistas con docentes y grupos focales con estudiantes de grados 10 y 11 de media vocacional; como resultado se generó una propuesta de arquitectura funcional que permite la generación de recomendaciones iniciales de contenidos administrados según el desempeño de los estudiantes y las características propias del territorio.

Palabras claves: Sistema de recomendación, plataforma de aprendizaje, modelo de datos, aprendizaje automático, educación.

\footnotetext{
${ }^{*}$ Para el desarrollo de esta investigación se contó con el apoyo técnico y financiero del Centro de Desarrollo Tecnológico CreaTIC. La participación activa de esta organización fue fundamental para el cumplimiento de los alcances del proyecto y posterior difusión de los resultados.

** Correo electrónico: andrea.otero@ clustercreatic.com

*** Correo electrónico: ceo@ clustercreatic.com

${ }^{* * * * *}$ Correo electrónico: camilo.pedraza@ clustercreatic.com

***** Correo electrónico: raul.canay@usc.es 


\section{ABSTRACT}

The research presents an approach based on data analytics and automatic learning mechanisms integrated into one of the most widely used digital learning platforms in the world (open EDX) as a contribution to the improvement of learning processes in middle school students in municipalities of Colombia. Methodologically, according to Creswell y Plano Clark (2007), and Clements et al. (2017) a system was created to recommend educational contents adapted to the individual characteristics of students considering the limitations in the use of technologies in educational institutions; we developed interviews with teachers and focus groups with students of $10^{\circ}$ and $11^{\circ}$ grade; as a result, a functional architecture proposal was generated that allows the generation of initial recommendations of contents managed according to the students' performance and the characteristics of the territory.

Keywords: Recommendation system, learning platform, data model, machine learning, education.

\section{Introducción}

Las tecnologías de información y comunicaciones (TIC) han propiciado el acceso a tipos de información con gran cantidad de información generada a partir de internet o de centros de datos específicos, siendo esta información diversa por los diferentes dispositivos por los cuales se puede acceder (Tablet, Smartphone, computadores, ebooks, smartwatches) (Ramírez-Montoya y García-Peñalvo, 2017). Esto ha permitido que el sector educativo inicie un proceso de transformación digital dejando a un lado sus procesos tradicionales de enseñanza y aprendizaje, superando limitaciones y obstáculos que impedían el acceso como la limitante geográfica o los tiempos del esquema tradicional educativo (Avello Martínez y Duart, 2016). De acuerdo con esto, las tendencias en el sector educativo como plataformas en línea, cursos abiertos masivos (MOOC), gestores de contenidos de aprendizaje y el e-learning constituyen herramientas utilizadas hoy en día para mejorar calidad en la educación.

Según el Foro "Invertir en jóvenes como condición para una paz duradera en Colombia" (UNFPA Colombia, 2016) realizado por el Fondo de Población de las Naciones Unidas en Julio de 2016, en Colombia, cerca de 13 millones de personas se encuentran en el rango 14 - 28 años, constituyendo el 27\% de la población colombiana. El gasto total (nacional y territorial) en adolescencia y juventud equivale al $2.9 \%$ del PIB. El sector que destina mayores recursos específicamente para jóvenes es educación (40,8\% del presupuesto nacional). A pesar de que la educación es el sector donde más 
TIC para la educación: sistema adaptativo basado en mecanismos de aprendizaje automático para la apropiación de tecnologías en estudiantes de educación media

se invierte, aún subsisten grandes brechas en el acceso y la calidad de la educación entre los jóvenes. Aquellos que viven en zonas rurales acceden un $24 \%$ menos a educación secundaria y media que los de zonas urbanas. $13,5 \%$ de estudiantes no terminan la primaria y $30,9 \%$ de adolescentes no terminan la secundaria. $13 \%$ de los adolescentes que terminan la secundaria no continúan en grado $10^{\circ}$ y el $11,3 \%$ de quienes presentan pruebas SABER $11^{\circ}$ no ingresan a educación superior (Departamento Nacional de Planeación, 2014)

En este panorama surge la necesidad de elaborar una propuesta orientada a la incorporación de TIC en el aula de clase que fomente la capacidad transformadora de los niños (Ministerio TIC, 2016), el desafío de estructurar tal programa dirigido a estudiantes de educación media surgió con dos objetivos específicos: i) Estudiar los referentes teóricos y pragmáticos alrededor de la inclusión de herramientas tecnológicas en el aula de clase y ii) Proponer una plataforma que coadyuve a la gestión de contenidos a partir de las necesidades reales de los niños, niñas y jóvenes. Lo anterior a partir de un ejercicio piloto a desarrollar en el departamento del Cauca.

El proceso inició con una revisión literaria alrededor de los procesos de incorporación de tecnologías de la información y las comunicaciones -TIC- en el aula de clase, así como de las dinámicas y las posibilidades de fomento al proceso de aprendizaje en los niños desde las instituciones educativas. Se revisaron, además, experiencias internacionales en estos temas a fin de contar con referentes teóricos y empíricos para avanzar en la estructuración de una propuesta sustentada desde lo técnico y pertinente desde un enfoque territorial.

Más allá de prepararlos para un mercado laboral cada vez más imbricado con la tecnología, la incorporación de tecnología permite a los niños desarrollar competencias de pensamiento lógico, resolución de problemas complejos, recursividad y coherencia narrativa. La propuesta que se realiza, posibilita la gestión de contenidos en ambientes reales y conectados con el entorno de los estudiantes a fin de optimizar y facilitar el acceso a contenidos de calidad y pertinentes.

\section{Revisión literaria}

Para avanzar en los objetivos de la investigación, se dividió la revisión literaria en tres componentes que posibilitan el análisis de referentes. En primera instancia se revisan autores cuya producción intelectual de relevancia se concentre en las relaciones estudiante-educador y el establecimiento de estrategias de monitoreo.

En segundo lugar, se abordan elementos concernientes a la evaluación de técnicas, algoritmos y modelos para realizar recomendaciones en el proceso de 
enseñanza y aprendizaje. Finalmente se analizan los Sistemas de recomendación que trabajen con la plataforma de Open EDX.

Relación estudiante - educador: Usan técnicas de minería de datos con el fin de analizar patrones entre el uso de la tecnología y las experiencias de aprendizaje de los estudiantes, determinando de esa forma la ruta de aprendizaje de cada docente (Howard et al., 2016). Otros autores proponen una herramienta que es capaz de relacionar a los estudiantes con supervisores teniendo en cuenta a la experiencia, carga máxima de trabajo e interés de este último con respecto a las preferencias del estudiante (Sanchezanguix et al., 2019)

En los trabajos de autores como Pereira et al. (2018) y Birjali et al. (2018) extraen el perfil y preferencias de los estudiantes a través de redes sociales, con base en esta información realizan recomendaciones. Por otro lado, autores como Tahmasebi et al., (2018) obtienen información de los sitios web OCW visitados, de ahí extraen metadatos que relacionan con los estilos de aprendizaje dados por la teoría de Felder y Silverman (1988) para realizar recomendaciones. Los autores Bradac y Walek (2017), Udupi et al., (2016) y Cuong et al (2018) integran modelos de lógica difusa y web semántica para hacer más efectivo la adaptación de contenido a plataformas LMS. Los trabajos de Nye (2015) y Klašnja-Milićević et al. (2018) se enfocan en sistemas de recomendación basados en las necesidades y nivel de conocimiento de los estudiantes.

Acosta et al., (2018) elabora un modelo de data warehouse sobre el cual implementan técnicas de minería de datos para determinar el perfil de los estudiantes de acuerdo a su desempeño académico, factores socioeconómicos, demográficos y actitudinales. Por otro lado, para determinar el perfil de un estudiante, Duque Méndez et al., (2018) implementan un sistema de recomendación basado en contenido donde a través del test de CHAEA obtienen el estilo de aprendizaje del estudiante, de esa forma determinan y recomiendan actividades educativas adaptadas a su perfil. 
TIC para la educación: sistema adaptativo basado en mecanismos de aprendizaje automático para la apropiación de tecnologías en estudiantes de educación media

Evaluación de técnicas, algoritmos y modelos: Li et al., (2018) evalúan el desempeño del árbol de Naïve Bayes con el fin de determinar el estilo de aprendizaje de los estudiantes. Por otra parte, Núñez-Valdez et al., (2018) realizan una evaluación de doce (12) algoritmos populares dentro del aprendizaje automático: árboles de clasificación y regresión, redes neuronales artificiales y algoritmos para determinar la similitud, con el fin de construir un sistema de recomendación de libros electrónicos concebido como una tarea de regresión.

Por otra parte, Guanín et al., (2019) evalúan catorce (14) algoritmos de aprendizaje supervisado divididos en tres categorías: Crisp Rule Learning, Decision Trees y Evolutionary crisp rule learning, donde se obtiene un conjunto de reglas para la toma de decisiones y estrategias focalizadas en grupos de interés. Trifa et al., (2019) proponen evaluar el proceso de personalización de una plataforma de e-learning que esté acorde con el perfil de cada estudiante mediante un sistema referencial inteligente basado en agentes, específicamente en el agente Mod-Knowledge.

Sistemas de recomendación utilizando Open EDX: Los autores desarrollan un sistema de recomendación de recursos educativos para la plataforma EDX (Collado Sánchez, 2014), para este diseñan un algoritmo de filtrado colaborativo basado en puntuaciones de problemas obtenidos a través de EDX por cada uno de los compañeros de un curso; el algoritmo busca a los compañeros con patrones similares, es decir, los que tienen más problemas aprobados, luego se realiza las predicciones en función de la popularidad de dichos problemas. Por otra parte, Anacona et al., (2017) presentan una propuesta arquitectónica para descubrir patrones de navegación de los estudiantes dentro de los $\mathrm{MOOC}^{1}$, que soporta la plataforma EDX, mediante el modelo de espacio vectorial y el algoritmo LexRank con Umbral. Finalmente, Gope y Kumar Jain, (2018) proponen un sistema de recomendación basado en los requerimientos y estilos de

\footnotetext{
${ }^{1}$ Massive Open Online Courses, son una propuesta para llevar la educación de forma gratuita y de calidad.
} 
Andrea Otero, Wilfred Rivera, Camilo Pedraza y José Raúl Canay

Telos Vol. 21, No. 3 (2019). 526-543

aprendizaje, estos últimos son una versión adaptada de la teoría de Felder Silverman y funcionan exclusivamente para los MOOCs de edx.

\section{Metodología}

La investigación partió de un enfoque metodológico mixto, que involucra el uso de herramientas y técnicas cuantitativas y cualitativas.

Se realizó una recolección y análisis de datos que, combinados, permiten llegar a un mejor entendimiento del problema de investigación (Creswell y Plano Clark, 2007). Esta aproximación es ampliamente utilizada en las ciencias sociales (Pereira Perez, 2011) y muy útil para la investigación en educación pues posibilita una comprensión profunda de los fenómenos en observación (Sabino, 2003). Las entrevistas de tipo cualitativo se realizaron entre septiembre de 2018 y enero de 2019 a 10 expertos regionales. Usando técnicas de muestreo dirigido o intencional se seleccionaron las unidades elementales de la población según el juicio de los investigadores, dado que las unidades seleccionadas gozan de representatividad (Hernandez Sampieri et al., 2010) en virtud de la experiencia de los entrevistados como docentes (5 personas) durante más de 10 años, y conocedores de política pública (5 personas) dado que han sido Secretarios Municipales de Educación en los municipios de Popayán, Argelia, El Tambo, o que han desarrollado programas de incorporación de TIC en el aula de clase con la Corporación CreaTIC.

En el plano instrumental, se practicó la metodología de views and beyond (Clements et al., 2017) como una base para la construcción y documentación de la arquitectura, en la cual se establecieron los elementos, sus relaciones e interfaces para el desarrollo de una plataforma adaptativa. Por otra parte, con base en Guerrero Cordoba et al., (2016) el modelo de recomendación se obtiene mediante 6 pasos: 1. Colección de datos, 2. Preparación de datos, 3. Elección del modelo, 4. Entrenamiento, 5. Evaluación y finalmente, 6. Predicción. 
TIC para la educación: sistema adaptativo basado en mecanismos de aprendizaje automático para la apropiación de tecnologías en estudiantes de educación media

\section{Resultados y Discusión}

\section{Aproximación arquitectónica}

La propuesta arquitectónica contiene entre sus componentes la plataforma Open EDX, de ahora en adelante EDX, como plataforma encargada de administrar los estudiantes y contenidos educativos a los que pueden acceder. En la Figura 1, se puede observar su estructura.

Figura 1. Arquitectura de Open EDX.

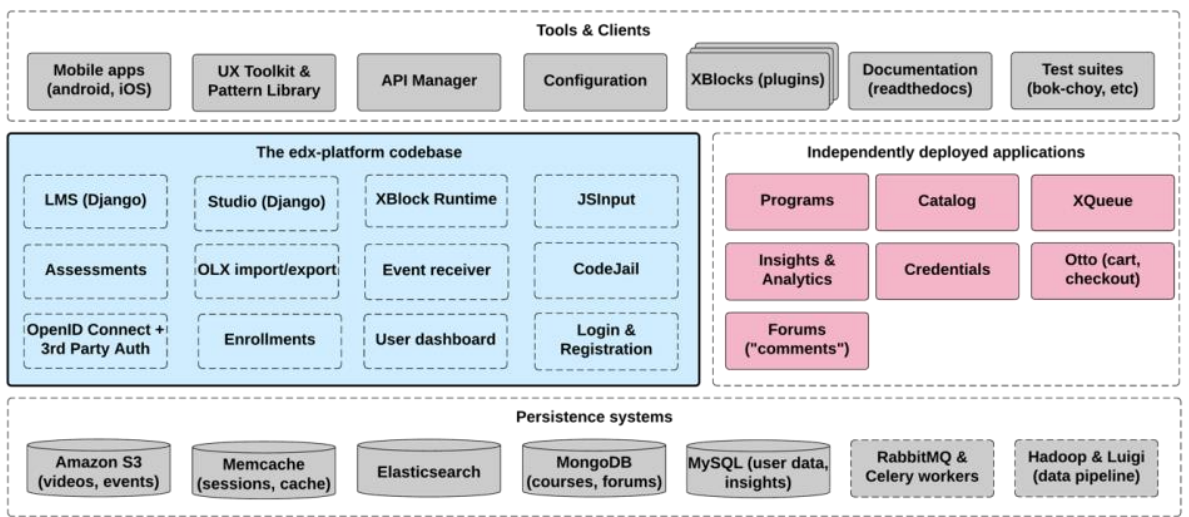

Fuente: Tomado de la página oficial de Open EdX (Open edX Developer's Guide, 2018).

EDX consta de varios componentes y está basado en una arquitectura de servicios para poder ser escalable (Collado Sánchez, 2014), lo que indica que sus componentes se pueden ejecutar de forma independiente y se puede ampliar si es necesario. El centro de su arquitectura es edx-platform, el cual contiene las aplicaciones de gestión de aprendizaje (LMS y creación de cursos (studio). Finalmente, utiliza dos gestores de base de datos: 1) MongoDB, un sistema de base de datos no relacional (NoSQL), en edx almacena el contenido de los cursos, debates y foros de discusión. 2)SQLite-MySQL, en entornos de desarrollo es usado SQLite, en producción MySQL, en este sistema de base de datos se almacena los datos de registro de los usuarios, inscripciones a cursos, el progreso del estudiante, el estado, entre otros. 
En ese sentido, en la Figura 2 se presenta la arquitectura propuesta para la implementación de una plataforma adaptativa basada en mecanismos de aprendizaje automático haciendo uso de edx.

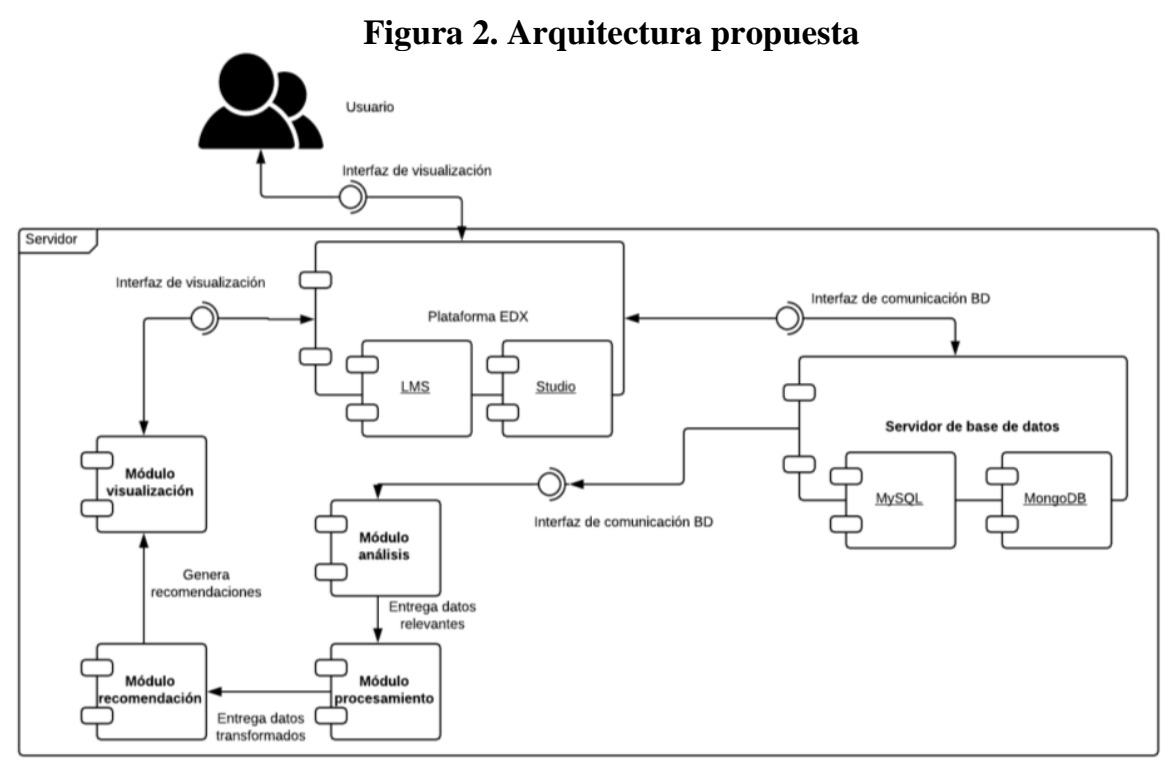

Fuente: Elaboración propia.

Como se puede observar en la Figura 2, la arquitectura consta de 6 componentes: Plataforma EDX, servidor de base de datos, módulos de análisis, procesamiento, recomendación y visualización.

En ese sentido, un usuario ingresa a la plataforma EDX donde puede visualizar la información de los cursos y formarse en alguno de ellos. Toda la información proporcionada por el usuario: su registro, inscripción en cursos, y el avance de aprendizaje en cada uno de ellos es almacenado en las bases de datos que se encuentran dentro del servidor de bases de datos. Finalmente, los datos almacenados son analizados 
TIC para la educación: sistema adaptativo basado en mecanismos de aprendizaje automático para la apropiación de tecnologías en estudiantes de educación media

y procesados por cada uno de los siguientes módulos para finalmente obtener una recomendación apropiada para el usuario y que este pueda visualizarla. Ahora bien, el flujo del funcionamiento de toda la plataforma se visualiza en la Figura 3, seguido a esto se explica cada uno de los módulos en más detalle.

\section{Figura 3. Flujo de funcionamiento de la plataforma adaptativa.}

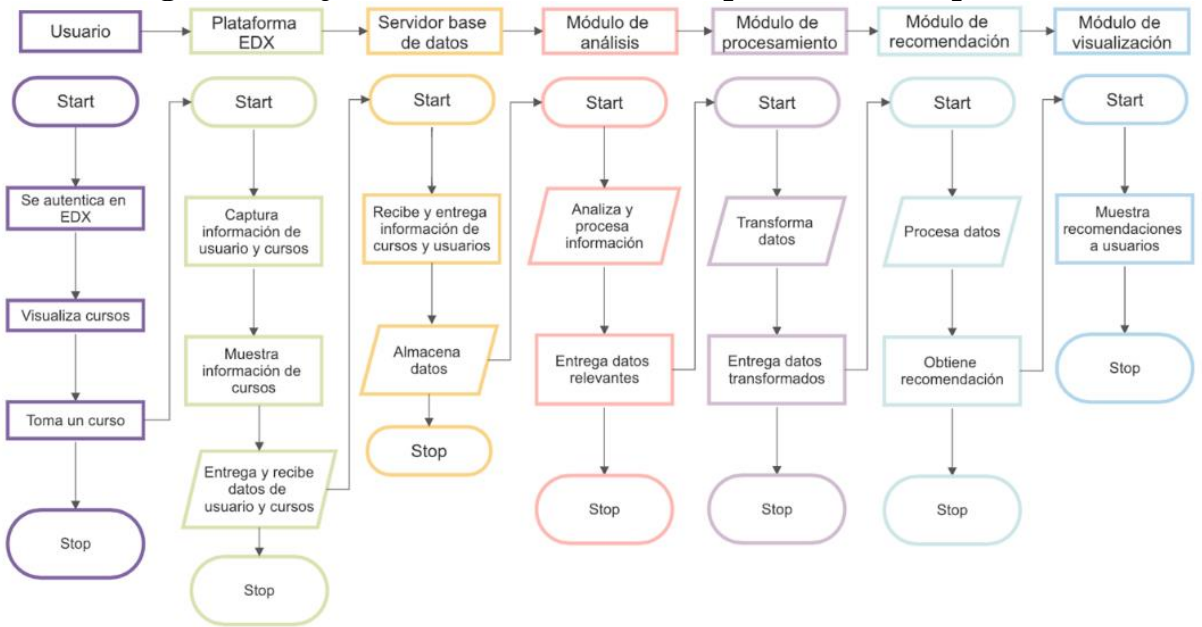

Fuente: Elaboración propia.

Ahora bien, el módulo de la plataforma EDX y el servidor de base de datos hacen parte de la arquitectura propia de EDX por lo que no se ahondará en detalles en estos.

\section{Plataforma EDX}

Es el medio por el cuál un usuario es capaz de visualizar la información de los cursos educativos y las recomendaciones realizadas por el sistema.

\section{Servidor de base de datos}

La información tanto de cursos como la de los usuarios de EDX es almacenada en dos gestores de bases de datos: MySQL y MongoDB. 


\section{Módulo de análisis}

Obtiene la información del servidor de base de datos, recorre los modelos de datos que proporciona cada uno de los gestores de bases de datos (MySQL y MongoDB) y finalmente selecciona los datos más relevantes. Para este caso, los más relevantes son los que se encuentran dentro de una base de datos o colección llamada edxapp para ambos gestores. En ese sentido, los datos específicos seleccionados se listan a continuación:
a. modulestore: Colección de la base de datos edxapp de MongoDB.
b. student_courseenrollment: Tabla de la base de datos edxapp de MySQL.
c. courseware_studentmodule: Tabla de la base de datos edxapp de MySQL.

\section{Módulo de procesamiento}

Después que el componente de análisis entrega la información relevante, se realiza el proceso de ETL (Extracción, transformación y carga) para los datos y posteriormente es realizada una técnica de preparación de los datos para la generación de una vista minable que pueda ser utilizada por el modelo definido generando resultados mostrados por el módulo de visualización para toma de decisiones.

El proceso de ETL de los datos se resume en las siguientes actividades:

a.Extracción: Conectarse a una fuente de datos, seleccionar y leer los datos relevantes

b. Transformación: Hacer operaciones sobre los datos

C. Carga: Guardar el resultado en un destino

\section{Módulo de recomendación}

Una vez el componente de procesamiento envía los datos transformados, el módulo de recomendación los recibe para conformar un conjunto de datos, llamado dataframe, que serán analizados por un algoritmo de aprendizaje automático y así poder entregar las recomendaciones correspondientes. 
TIC para la educación: sistema adaptativo basado en mecanismos de aprendizaje automático para la apropiación de tecnologías en estudiantes de educación media

Después de obtener los datos y conformar el dataframe, se crea adicional un conjunto de datos llamados trainset que será el conjunto de datos utilizado para entrenar el modelo de recomendación y hacerlo cada vez más preciso. Para ello se utiliza la técnica llamada factorización de matrices (Galarza Hernández, 2017), la cual se realizó implementando el algoritmo SVD (Descomposición en valores singulares).

Finalmente se aplica un proceso de validación cruzada o cross validation para mejorar los parámetros del algoritmo de predicción SVD y obtener recomendaciones más óptimas.

El algoritmo SVD y cross validation fueron implementados a través de las librerías surprise (Surprise documentation, 2015) y scikit-learn (scikit-learn documentation, 2018) para python.

En ese sentido, el modelo de recomendación desarrollado se presenta en la

Figura 4

Figura 4. Modelo de recomendación.

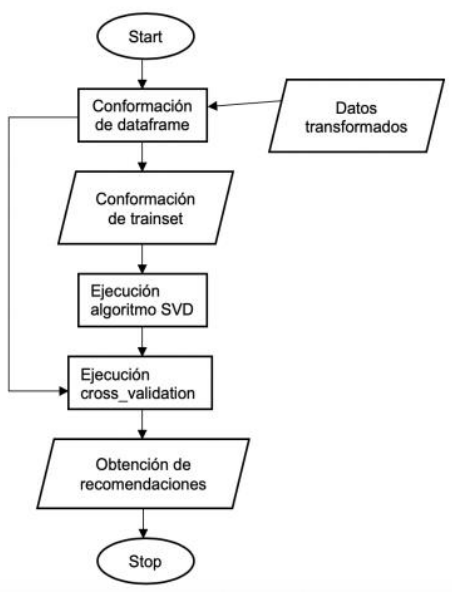

Fuente: Elaboración propia

\section{Módulo de visualización}

Para efectos de pruebas, se crea un proyecto utilizando el lenguaje de programación javascript, donde se puede observar las recomendaciones. Por lo tanto, 
se obtiene la información de los usuarios y cursos de la base de datos a través de un api que realiza la conexión a estas; luego, dicha api genera una URL por la cual la interfaz de javascript consulta las predicciones de acuerdo al usuario registrado, lo anterior se realiza mediante el protocolo http.

Finalmente, se crean 2 usuarios y 3 cursos de pruebas en la plataforma EDX. Uno de los usuarios es estudiante de último semestre de ingeniería de sistemas de la Universidad del Cauca, el segundo es ingeniera en electrónica y telecomunicaciones de la misma universidad. Cada uno de ellos realizó el proceso completo: entrar a la plataforma, registrarse, luego mirar los cursos e inscribirse en cada uno de ellos y finalmente realizar un avance en cada uno, de los cuales se tomó registro para entender de qué forma y en qué lugar la plataforma almacenaba la información.

Al ejecutar el modelo de recomendación con los datos obtenidos de los usuarios, se tiene como resultado una precisión de $89 \%$, una media de 1.18 y una desviación estándar de 0.36 .

\section{Resultados y discusión}

De acuerdo a los resultados obtenidos en el modelo de recomendación de la arquitectura propuesta, este presenta un error absoluto medio de 0.8198 , lo que quiere decir que el 81,98\% de las recomendaciones fueron útiles, lo que indica que el modelo implementado es prometedor.

Los expertos regionales en el ámbito TIC y educación manifiestan que las aplicaciones disponibles en la Web y los AppStore conllevan de manera ineluctable nuevos cambios sociales propios de la globalización, ciertos docentes se sienten inmigrantes digitales y enfrentan el desafío de introducirse en el entorno digital en el cual los estudiantes ya están inmersos; en la mayoría de los casos, los docentes son receptivos frente al uso de estas tecnologías siempre y cuando sean involucrados en los procesos de apropiación y encuentren los mecanismos a través de los cuales sus 
TIC para la educación: sistema adaptativo basado en mecanismos de aprendizaje automático para la apropiación de tecnologías en estudiantes de educación media

contenidos y metodologías se articulen con el uso de nuevas tecnologías. Si bien es inevitable que el uso de contenidos digitales genere cambios en los procesos actuales, en la medida en que el uso de plataformas y contenidos posibilite la participación activa del docente su integración tendrá un mayor nivel de efectividad.

Existe consenso en el ámbito literario alrededor de la necesidad de descubrir en los estudiantes los intereses y motivaciones intrínsecas para lograr un aprendizaje significativo; en esta vía se aumenta el deseo de aprender y se incrementan los niveles de eficacia que son materia de análisis y promoción por parte de la política pública. Una revisión de las plataformas y contenidos disponibles de manera gratuita evidencia la existencia de formas interactivas y didácticas de enseñar que atraen la atención de estudiantes mas no garantizan continuidad en el proceso.

\section{Conclusiones y trabajo futuro}

La plataforma Open EDX es una de las más utilizadas para enseñar y aprender de forma virtual, es una plataforma de código abierto que puede ser utilizada en cualquier parte del mundo. En ese sentido EDX, es capaz de almacenar gran cantidad de información correspondientes a estudiantes y educadores, es así como se hace una herramienta clave en el momento de analizar datos para contribuir en la mejora de un sistema educativo virtual, con dichos datos es posible reconocer las necesidades de los estudiantes, sus gustos y de esa forma realizar predicciones que lo impulsen a potenciar sus capacidades, de la misma forma con los educadores, al conocer las necesidades de sus estudiantes tomará acciones y decisiones correctas acerca de su metodología de enseñanza. De esa manera, la propuesta de un sistema adaptativo basado en mecanismos de aprendizaje automático abre las puertas para seguir mejorando el entorno e-learning. Queda como trabajo futuro mejorar el modelo de recomendación y adaptar una aplicación dentro de la plataforma EDX para que sea de más fácil acceso a los estudiantes poder visualizar sus recomendaciones. 
En la educación, la influencia tecnológica ha cambiado el paradigma de la enseñanza comprometiendo a docentes diversificar sus prácticas y replantear procesos didácticos en función de las tecnologías de la información y comunicaciones de manera que sean más efectivos y eficientes. Pese a esto, aún se encuentran limitaciones en los mecanismos para proporcionar los recursos educativos. Los estudiantes reciben información que no va acorde con sus características individuales, estilos de aprendizaje y preferencias; además que no se tiene en cuenta los recursos tecnológicos disponibles en su entorno, tales como un computador, Tablet, Smartphone, Smart TV, dispositivos para desarrollar proyectos IoT (internet de las cosas) o aprender programación, entre otros, evidenciando la necesidad de contar con un sistema, plataforma y/o aplicación que facilite recursos educativos, aprendiendo de la experiencia de los estudiantes para tomar decisiones más acordes con su perfil de aprendizaje.

Recientes estudios han demostrado la aplicación de mecanismos de aprendizaje automático para la identificación y recomendación de contenidos a usuarios en diferentes contextos, siendo usados para construir sistemas que mejoren en función de la experiencia lo que los lleva a tomar mejores decisiones e identificando apropiadamente la información o contenidos que se presentan a cada usuario. En el ámbito del territorio caucano, el modelo propuesto presenta múltiples aplicaciones en virtud de sus posibilidades de mejoramiento de la calidad en la educación.

Actualmente, son múltiples las aplicaciones disponibles para el fomento de la educación y las TIC en ámbitos como matemáticas, ciencias, lenguaje, manejo de idiomas, entre otras. Sin embargo, pocas son las herramientas que personalicen u orientes sus esfuerzos hacia el mejoramiento de los estudiantes en virtud de su desempeño. Si bien algunas plataformas han mostrado su eficacia en términos de seguimiento a los estudiantes, disponibilidad de contenidos, facilidad de acceso y amplia oferta recursos transmedia, no se han detectado casos atemperados a la realidad latinoamericana que permitan un seguimiento a los estudiantes en términos de su 
TIC para la educación: sistema adaptativo basado en mecanismos de aprendizaje automático para la apropiación de tecnologías en estudiantes de educación media

desempeño y que logre dinamizar la plataforma poniéndola al servicio real de los estudiantes. Si se analiza este elemento, la solución tecnológica termina por simular el trabajo de un docente que en la práctica adecua sus contenidos, metodologías y enfoque de trabajo a las realidades de cada contexto y, para el caso de docente sobresalientes, a la particularidad de cada estudiante.

La realidad en el ámbito colombiano y latinoamericano no es diferente. Este tipo de soluciones tecnológicas posibilita el acceso y pertinencia de los contenidos educativos activando un sistema de recomendaciones que se nutre a partir de su uso. $\mathrm{Si}$ bien la labor del docente seguirá siendo irremplazable por varias décadas, la tecnología comienza a transformar los mecanismos con los cuales se aprende, permitiendo a los estudiantes acceder a contenidos atemperados a sus capacidades, evitando frustraciones que resultan catastróficas y socavan las posibilidades de crecimiento y desarrollo por parte de los estudiantes.

\section{Referencias Bibliográficas}

Acosta, Julio César., La Red Martínez, David. y Primorac, Carlos. (2018). Determinación de Perfiles de Rendimiento Académico En La UNNE Con Minería de Datos Educacional Facultad de Ciencias Exactas y Naturales y Agrimensura. In XX Workshop de Investigadores En Ciencias de La Computación (WICC 2018, Universidad Nacional Del Nordeste). Corrientes, Argentina.

Anacona, Fabian., Solarte, Mario. y Ramírez, Gustavo. (2017). Discovery of Navigation Patterns in Open EdX - An Architectural Approach. Revista Ingeniería e Innovación RIINN. Volumen 5, No. 1. Colombia (Pp. 43-50).

Avello Martínez, Raidell., y Duart, Josep M. (2016). Nuevas Tendencias de Aprendizaje Colaborativo En E-Learning: Claves Para Su Implementación Efectiva. Estudios Pedagógicos. Volumen 42, No. 1. Chile (Pp. 271-82).

Birjali, Marouane., Beni-Hssane, Abderrahim. y Erritali, Mohammed. (2018). A Novel Adaptive E-Learning Model Based on Big Data by Using Competence-Based Knowledge and Social Learner Activities. Applied Soft Computing. Volumen 69. Marruecos (Pp. 14-32).

Bradac, Vladimir. y Walek, Bogdan. (2017). A Comprehensive Adaptive System for ELearning of Foreign Languages. Expert Systems with Applications. Volumen 90. República Checa (Pp. 414-26).

Clements, Paul., Bachmann, Felix., Bass, Len., Garlan, David., Ivers, James., Little, Reed., Merson, Paulo., Nord, Robert. y Stafford, Judith. (2017). Documenting 
Software Architecture Views and Beyond (Segunda edición). Editorial Addison-Wesley. Estados Unidos.

Collado Sánchez, Alma. (2014). Sistema de Recomendación de Recursos Basado En Filtrado Colaborativo Para La Plataforma EdX. Proyecto fin de carrera. Ingeniería técnica de telecomunicaciones, especialidad en telemática. Universidad Carlos III de Madrid. España.

Creswell, John W. y Plano Clark, Vicki L. (2007). Designing and Conducting Mixed

Methods Research. Editorial SAGE Publications. Estados Unidos.

Cuong, Nguyen Dinh Hoa., Arch-Int, Ngamnij. y Arch-Int, Somjit. (2018). FUSE: A

Fuzzy-Semantic Framework for Personalizing Learning Recommendations.

International Journal of Information Technology \& Decision Making.

Volumen 17. No. 04. Tailandia (Pp. 1173-1202).

Departamento Nacional de Planeación. (2014).Reporte. Conpes 173: Lineamiento Para

La Generación de Oportunidades Para Los Jóvenes. Bogotá D.C., Colombia.

Duque Méndez, Néstor Darío., Rodríguez Marín, Paula A. y Ospina Herrán, Alejandra.

(2018). Recomendación de Estrategias de Aprendizaje Personalizadas Basadas

En El Test de CHAEA Recommendation of Customized Learning Strategies

Based on the CHAEA. Scientia et Technica. Volumen 23. No. 02. Colombia (Pp. 222-29).

Felder, Richard M. y Silverman, Linda K. (1988). Learning and Teaching Styles in Engineering Education. Engineering Education. Volumen 78. Estados Unidos (Pp. 674-81).

Galarza Hernández, Javier. (2017). Reducción de Dimensionalidad En Machine

Learning.Tesis de grado. Título de grado en Ingeniería de Tecnologías y

Servicios de Telecomunicaciones. Universidad Politecnica de Valencia. España.

Gope, Jyotirmoy. y Jain, Sanjay Kumar. (2018). A Learning Styles Based Recommender System Prototype for EdX Courses. International Conference

On Smart Technology for Smart Nation, SmartTechCon 2017. Bengaluru, India.

Guanín, Jorge., Díaz, Raúl. y Oviedo, Byron. (2019). Descubrimiento de Reglas de Clasificación Para Estudiantes Que Se Inscriben Del Bachillerato a Carreras Universitarias. Universidad y Sociedad. Volumen 11. No. 2. Ecuador (Pp. 220-26).

Guerrero Cordoba, Fredy Mauricio., Otero Cano, Andrea., Duran Dorado, Diego Fabian. y Arciniegas Herrera, Jose Luis. 2016. Método Computacional Para La Identificación de Funciones de Pertenencia En Entornos de Lógica Difusa. 2016 IEEE 11th Colombian Computing Conference (CCC). Popayán, Colombia.

Hernández Sampieri, Roberto., Fernandez Collado, Carlos. y Baptista Lucio, Maria del 
TIC para la educación: sistema adaptativo basado en mecanismos de aprendizaje automático para la apropiación de tecnologías en estudiantes de educación media

Pilar. (2010). Metodolodía de La Investigación (Quinta edición). Editorial McGraw-Hil. México

Howard, Sarah K., Ma, Jun. y Yang, Jie. (2016). Student Rules: Exploring Patterns of Students' Computer-Efficacy and Engagement with Digital Technologies in

Learning. Computers and Education. Volumen 101. Australia (Pp. 29-42). Klašnja-Milićević, Aleksandra., Ivanović, Mirjana., Vesin, Boban. y Budimac, Zoran. (2018). Enhancing E-Learning Systems with Personalized Recommendation Based on Collaborative Tagging Techniques. Applied Intelligence. Volumen 48. No. 6. Serbia (Pp. 1519-1535).

Li, Ling Xiao. y Abdul Rahman, Siti Soraya. (2018). "Students ' Learning Style Detection Using Tree Augmented Naive Bayes.” Royal Society Open Science. Extraído de https://royalsocietypublishing.org/doi/10.1098/rsos.172108 consulta 05/01/2019.

Ministerio TIC. (2016).Informe. Informe de Gestión Al Congreso de La República 2015. Ministerio TIC Colombia. Colombia.

Núñez-Valdez, Edward Rolando., Quintana, David., González Crespo, Ruben., Isasi, Pedro. y Herrera-Viedma, Enrique. (2018). A Recommender System Based on Implicit Feedback for Selective Dissemination of Ebooks. Information Sciences. Volumen 467. España (Pp. 87-98).

Nye, Benjamin D. (2015). Intelligent Tutoring Systems by and for the Developing World: A Review of Trends and Approaches for Educational Technology in a Global Context. International Journal of Artificial Intelligence in Education. Volumen 25. No. 2. Estados Unidos (Pp. 177-203).

Open edX Developer's Guide. (2018). Open EdX Architecture. Extraído de https://edx.readthedocs.io/projects/edx-developerguide/en/latest/architecture.html consulta 05/02/2019.

Pereira, Crystiam Kelle., Campos, Fernanda., Ströele, Victor., David, José Maria N. y Braga, Regina. (2018). BROAD-RSI - Educational Recommender System Using Social Networks Interactions and Linked Data. Journal of Internet Services and Applications. Volumen 9. No. 7. Brasil (Pp. 1-28).

Pereira Perez, Zulay. (2011). Los Diseños de Método Mixto En La Investigación En Educación : Una Experiencia Concreta Mixed Method Designs in Education Research : A Particular Experience. Revista Electrónica Educare. Volumen 15. No. 1 Costa Rica (Pp. 15-29).

Ramírez-Montoya, María Soledad. y García-Peñalvo, Francisco José. (2017). Presentación. La Integración Efectiva Del Dispositivo Móvil En La Educación y En El Aprendizaje. RIED. Revista Iberoamericana de Educación a Distancia. Volumen 20. No 2. México (Pp. 29-47).

Sabino, Carlos. (2003). EI Proceso de Investigación. Editorial Panapo, Venezuela. Sanchez-anguix, Victor., Chalumuri, Rithin. y Julian, Vicente. (2019). A MultiObjective Evolutionary Proposal for Matching Students to Supervisors. Springer International. Volumen 800. España (Pp. 94-102). 
scikit-learn documentation. (2018). Sklearn.Model_selection.Cross_validate. Extraído de https://scikit-learn.org/stable/modules/cross_validation.html\#crossvalidation consulta 06/02/2019.

Surprise documentation. (2015). Matrix Factorization-Based Algorithms. Extraído de https://surprise.readthedocs.io/en/stable/matrix factorization.html consulta $06 / 02 / 2019$.

Tahmasebi, Mohammad., Ghazvini, Faranak Fotouhi. y Esmaeili, Mahdi. (2018). Implementation and Evaluation of a Resource-Based Learning Recommender Based on Learning Style and Web Page Features. Journal of Web Engineering. Volumen 17. No. 4. Irán (Pp. 284-304).

Trifa, Amal., Hedhili, Aroua. y Lejouad Chaari, Wided. (2019). Knowledge Tracing with an Intelligent Agent, in an e-Learning Platform. Education and Information Technologies. Volumen 24. No. 1. Túnez (Pp. 711-41).

Udupi, Prakash Kumar., Malali, Puttaswamy. y Noronha, Herald. (2016). Big Data Integration for Transition from E-Learning to Smart Learning Framework. 2016 3rd MEC International Conference on Big Data and Smart City (ICBDSC). Mascate, Omán.

UNFPA Colombia. (2016). Día Mundial de Población: Foro ‘Invertir En Jóvenes Como Condición Para Una Paz Duradera En Colombia.' Extraído de https://colombia.unfpa.org/es/noticias/día-mundial-de-población-foro"invertir-en-jóvenes-como-condición-para-una-paz-duradera-en consulta $01 / 04 / 2019$. 